\title{
The relationship between diet soda soft drinks, artificial sweeteners, and
}

\section{diabetic retinopathy: A cross-sectional study}

Hyder Osman Mirghani ${ }^{1}$, Naif Mamdouh Alali², Hani Basher Albalawi², Majed S. Alqahtani ${ }^{3}$, Iman Mohsen Abutaleb Qisi ${ }^{4}$, and Naif Khalf Almutairi ${ }^{5}$

1. Internal Medicine and Endocrine, Medical Department, Faculty of Medicine, University of Tabuk, Saudi Arabia

2. Ophthalmology Department, Faculty of Medicine, University of Tabuk, Saudi Arabia

3. Family Medicine Consultant, King Fahd Specialist Hospital, Ministry of Health, Tabuk, Saudi Arabia

4. Diabetes Educator, King Fahd Specialist Hospital, Ministry of Health, Tabuk, Saudi Arabia

5. Consultant Ophthalmologist and Vitreoretinal disease, Jeddah Eye Hospital, Jeddah, Saudi Arabia

\section{RESEARCH}

Please cite this paper as: Mirghani HO, Alali NM, Albalawi HB, Alqahtani MS, Qisi IMA, Almutairi NK. The relationship between diet soda soft drinks, artificial sweeteners, and diabetic retinopathy: $A$ cross-sectional study. AMJ 2020;13(5):174-178.

https://doi.org/10.35841/1836-1935.13.5.174-178

\section{Corresponding Author:}

Hyder Osman Mirghani

Faculty of Medicine, University of Tabuk, PO Box 3378

Tabuk 51941, Saudi Arabia

Email: s.hyder63@hotmail.com

\section{ABSTRACT}

\section{Background}

The relationship between diabetic retinopathy and dietdrink (soft soda), non-nutritive sweeteners have not been fully determined. We here attempted to determine it.

\section{Aims}

The study aimed to assess the association between soda soft drinks, non-nutritive sweeteners, and diabetic retinopathy.

\section{Methods}

A cross-sectional descriptive study was conducted at a diabetes center in Tabuk city, Saudi Arabia from September 2019 to April 2020: 174 patients with type 2 diabetes mellitus (DM) were interviewed using a structured questionnaire: demographic data, the DM duration, the lifestyles (exercise, smoking, and diet), and the medications. The weekly amount of diet soda and daily consumption of non-nutritive sweeteners were assessed. These were analysed with association with retinopathy.

Results

The glycated haemoglobin per cent was $8.50 \pm 1.60$, the diet soda and artificial sweeteners consumptions were $1.18 \pm 1.57$ cans/week and $0.89 \pm 1.26$ sachets/day, respectively. No association was found between artificial sweeteners, diet soda, and diabetic retinopathy (Wald, 1.251, and 0.213, P-values, 0.263, and 0.644, 95 per cent $\mathrm{Cl}$, 0.583-7.204, and 0.190-2.793 respectively). Retinopathy incidence was associated with the DM duration (Wald, 8.095, P-value, 0.004, and 95 per cent $\mathrm{Cl}, 0.766-0.952$ ).

\section{Conclusion}

Diet soda and non-nutritive sweeteners were not associated with diabetic retinopathy, irrespective of gender or body mass index. Diabetic retinopathy was associated with the duration of DM after its first diagnosis.

\section{Key Words}

Diabetic retinopathy, diet soda soft drinks, non-nutritive sweeteners

\section{What this study adds:}

\section{What is known about this subject?}

The literature assessing the relationship between diet soda and non-nutritive sweeteners on diabetic retinopathy are lacking. This is the first study to assess this important issue in Saudi Arabia

\section{What new information is offered in this study?}

The study found no association between diet soda, non- 
nutritive sweeteners, and diabetic retinopathy. 
3. What are the implications for research, policy, or practice?

Diet soda and non-nutritive sweeteners in moderate amount may not affect diabetic retinopathy.

\section{Background}

We are in the diabetes epidemic. Currently, 350 million are affected by this lifelong morbid disease, and the projection for the year 2030 is 438 million. Furthermore, 8 million people suffer from prediabetes and with every diagnosed patient, another person is undiagnosed, so the number is double. $^{1-4}$ The Kingdom of Saudi Arabia is among the countries with the highest prevalence (21.1 per cent). Patients with type 2 diabetes could present with microvascular complications including diabetes retinopathy at the time of diagnosis with deleterious consequences on the affected person, healthcare providers, and the community at large. People suffering from diabetes mellitus have three times the health expenditure compared to others without the disease mainly due to retinopathy. ${ }^{5,6}$

Worldwide in 2010, 32.4 million people are blind and 191 million visually impaired, from them 0.8 million were blind, and 3.7 million were visually impaired because of diabetes retinopathy, with an alarming increase of 27 per cent and 64 per cent, respectively. ${ }^{7}$ The prevalence of diabetic retinopathy in Saudi Arabia ranged from 19.7 per cent to 36.8 per cent in different regions. ${ }^{8-10}$ Non-nutritive sweeteners (Acesulfame-K, Aspartame, sucralose, and Cyclamate) are widely consumed in soft drinks and canned food and have been linked to various diseases including diabetes mellitus, obesity, and cancer. ${ }^{11}$

To our best of knowledge, no researchers have assessed the relationship of diet soda and NNSs to diabetic retinopathy in the Kingdom of Saudi Arabia, and it is a few worldwide types of research study that relationship. Thus we conducted this research, in the present study, we thought to assess the relationship of diet soda and non-nutritive sweeteners to diabetic retinopathy in Tabuk, Saudi Arabia.

\section{Method}

A cross-sectional hospital-based study was conducted in the diabetes center, King Fahd Specialist Hospital, Tabuk, Saudi Arabia during the period from September 2019 to April 2020; One hundred seventy-four participants with type 2 diabetes were enrolled in the study. Children with diabetes mellitus and pregnant ladies were not included.

\section{Study population and sampling:}

Subjects with the diagnosis of type 2 diabetes in King Fahd Specialist Hospital and are coming for regular visits were approached. Pregnant ladies, severely ill patients, patients with renal impairment and liver cirrhosis, and patients with malignancies were excluded. A face to face interview will be conducted using a structured questionnaire that inquires about socio-demographic data, duration since the diagnosis of type 2 diabetes, cigarette smoking, hypertension, physical exercise, and if following a healthy dietary habit. The medications used by the patient including lipid-lowering medication (may affect the development and progression of diabetes retinopathy), diabetic retinopathy also documented (consisted of funduscopy examination by using slit-lamp with $90 \mathrm{D}$ lenses and indirect ophthalmoscopy). DR was defined as the presence of one or more of the following lesions: microaneurysms, blot or flame-shaped haemorrhages, hard exudates, cotton-wool spots, or evidence of laser treatment for DR at baseline. DR was categorized as nonproliferative (NPDR) or proliferative (PDR), determined by the presence of retinal neovascularization.

The consumption of soda soft drinks was reported as follows: No consumption, <1 can of $350 \mathrm{ml} /$ week (mild), 1-4 cans (moderate), and > 4cans/week (high). ${ }^{11}$ The amount, duration, and frequency of non-nutritive sweeteners were also reported in sachets/tabs per day.

Weight, height, and Body Mass Index (BMI) as measured using the formula: Weight in $\mathrm{Kg} /$ height in (meters) were recorded. Obesity was defined as a BMI $\geq 30$, overweight 25-29, and $18-25$ as normal. The most recent glycated haemoglobin (HbA1c per cent) was collected from the patient's records to assess the degree of glycaemic control, and glycated haemoglobin of $<7$ was regarded as good glycaemic control. ${ }^{4}$ Written informed consent was signed by all the participants, and an approval letter was obtained from the ethical committee of the University of Tabuk (ref. number, UT-93-1-2020, date, 27/1/2020).

\section{Statistical analysis:}

Data were exported to the Statistical Package for Social Sciences for the analysis. Descriptive and summary statistics were carried out to describe study participants according to different characteristics and proportions were computed to find out the relationship between soda soft drinks, nonnutritive sweeteners, and diabetic retinopathy. A-P-value of $<0.05$ was considered significant. 


\section{Results}

There were 174 patients with diabetes (49.5 per cent women), their ages ranged from 25-94 years with a mean of $49.80 \pm 13.45$ years, the duration since the diagnosis of diabetes was $9.36 \pm 6.56$ years, the body mass index of the participants was $26.96 \pm 4.51$, the glycated haemoglobin was $8.50 \pm 1.60$, the diet soda and artificial sweeteners were $1.18 \pm 1.57$ cans/week and0.89 \pm 1.26 sachets/tabs/day respectively. In the present study, less than half (49.4 per cent) were adherent to regular exercise, family history of diabetes, hypertension, and coronary artery disease were reported in 71.3 per cent, 55.2 per cent, and 14.9 per cent respectively. Regarding medications, 37.9 per cent were on anti-hypertensive drugs, 23 per cent on lipid-lowering medications, 35.6 per cent were on oral hypoglycaemic drugs. 12.6 per cent were on insulin only, while 51.7 per cent were using both oral therapy and insulin combination. In the present study, nearly one third (31 per cent) suffer from diabetic retinopathy (both non-proliferative diabetic retinopathy and proliferative diabetic retinopathy), and 66.6 per cent were either overweight or obese Table 1.

In the current study, no association was found between artificial sweeteners, diet soda, and diabetic retinopathy (Wald, 1.251, and 0.213, P-values, 0.263, and 0.644, 95 per cent $\mathrm{Cl}, 0.583-7.204$, and $0.190-2.793$ respectively) which was associated with the duration of diabetes mellitus (Wald, 8.095, P-value, 0.004, and 95 per cent $\mathrm{Cl}, 0.766-0.952)$. Table 2 depicted the association of diabetic retinopathy and other patient's characteristics.

\section{Discussion}

Diet soda soft drinks and non-nutritive sweeteners are widely used for calorie restriction among patients with diabetes mellitus and high body mass index. However, literature assessing the relationship of soda soft drinks to diabetic retinopathy in the Kingdom are scarce. We found a single recent study ${ }^{12}$ that evaluated the relationship in Australia and found that high consumption of soda diet drinks is associated with the severity of diabetic retinopathy. A decreased additive sugar intake is recommended by many organizations, substitution with non-nutritive sweeteners is among strategies to achieve this goal. ${ }^{13}$ Previous studies from Australia found 18.2 per cent of the adult population consumed non-nutritive sweeteners, with the most common food sources being carbonated soft drinks, tabletop sweeteners, and yogurt and their benefit in energy reduction and weight loss are controversial. ${ }^{14}$ The association of non-nutritive sweeteners with diabetes, cardiovascular disease, obesity, depression, and cognitive impairment is inconclusive. ${ }^{11}$ NNSs interfere with learned responses that contribute to control glucose and energy homeostasis, interfere with gut microbiota and induce glucose intolerance, and interact with sweet-taste receptors expressed throughout the digestive system that plays a role in glucose absorption and trigger insulin secretion. ${ }^{15}$ The effects of non-nutritive sweeteners and diet soda on plasma sugar and glycaemic control is a matter of debate, while their effects on diabetic retinopathy were not studied in the Kingdom of Saudi Arabia. ${ }^{16,17}$

In the present study, artificial sweeteners and diet soda consumption were not associates with diabetic retinopathy. A study conducted in Australia ${ }^{18}$ found that diet soda is associated with proliferative diabetic retinopathy in contradiction to the present findings in which diet soda was not associated with diabetic retinopathy, another study from Australia $^{12}$ found the association of artificial sweeteners and the severity of diabetic retinopathy, a plausible explanation could be the lower amount of daily consumption of soda soft drinks and artificial sweeteners observed in the current sample and the fact that the majority of our patients had mild non-proliferative diabetic retinopathy. The current data showed that 47.1 per cent of patients with diabetes were consuming non-nutritive sweeteners and diet soda in line with Fenwick et al. ${ }^{18}$ who observed diet soda consumption in 46.8 per cent of patients. The current data showed that 66.6 per cent were obese or overweight and the BMI was not associated with diabetic retinopathy, similar findings were concluded by Sujanitha et al. $^{19}$ who found no association between diabetes complications and BMI, the association between diabetes complications and BMI seems to occur at levels $\geq$ 27.5 in particular among men, ${ }^{20}$ in the present study most patients BMI was less than 27.5. In the present study, diabetic retinopathy was associated with the duration of diabetes in similarity to previous observations, ${ }^{21,22}$ a study published in Hong $\mathrm{Kong}^{23}$ found no association between gender and diabetic retinopathy in line with the current observations.

The limitations of the study were the relatively small size of the study group and the study was conducted at a single diabetes center, so generalization to the whole Kingdom of Saudi Arabia cannot be insured.

\section{Conclusion}

Diet soda and non-nutritive sweeteners were not associated with diabetic retinopathy with no differences by gender or body mass index. Diabetic retinopathy was associated with the duration since the diagnosis of diabetes. 


\section{References}

1. Jia G, Aroor AR, Martinez-Lemus LA, et al. Overnutrition, mTOR signaling, and cardiovascular diseases. Am J Physiol Regul Integr Comp Physiol. 2014;307:R1198-206.

2. Maiese K. Novel nervous and multi-system regenerative therapeutic strategies for diabetes mellitus with mTOR. Neural Regen Res. 2016;11(3):372-85. doi: 10.4103/1673-5374.179032.

3. International Diabetes Federation, Middle East, and North Africa 2015.

4. American Diabetes Association Standards of Medical Care in Diabetes. Lifestyle Management Diabetes Care 2017;40(Suppl. 1):S33-S43. doi: 10.2337/dc17-S007

5. AL-Nozha MM, Al-Maatouq MA, Al-Mazrou YY, et al. Diabetes in Saudi Arabia. Saudi Med J 2004 Nov;25(11):1603-10

6. Alhowaish AK. Economic costs of diabetes in Saudi Arabia. J Family Community Med. 2013;20(1):1-7. doi: 10.4103/2230-8229.108174.

7. Leasher JL, Bourne RR, Flaxman SR, et al. Global estimates on the number of people blind or visually impaired by Diabetic Retinopathy: A Meta-analysis from 1990 to 2010. Diabetes Care. 2016;39(9):1643-9. doi: $10.2337 / \mathrm{dc} 15-2171$.

8. Al-Rubeaan K, Abu El-Asrar AM, Youssef AM, et al. Diabetic retinopathy and its risk factors in a society with a type 2 diabetes epidemic: a Saudi national Diabetes Registry-based study. Acta Ophthalmol. 2015;93(2):e140-7. doi: 10.1111/aos.12532. Epub 2014 Oct 1.

9. Hajar S, Al Hazmi A, Wasli M, et al. Prevalence and causes of blindness and diabetic retinopathy in Southern Saudi Arabia. Saudi Med J. 2015;36(4):449-55. doi: 10.15537/smj.2015.4.10371.

10. Al Ghamdi AH, Rabiu M, Hajar S, et al. Rapid assessment of avoidable blindness and diabetic retinopathy in Taif, Saudi Arabia. Br J Ophthalmol. 2012;96(9):1168-72. doi: 10.1136/bjophthalmol-2012-301874. Epub 2012 Jul 11.

11. Fenwick EK, Gan AT, Man RE, et al. Diet soft drink is associated with increased odds of proliferative diabetic retinopathy. Clin Exp Ophthalmol. 2018;46(7):767-776. doi: 10.1111/ceo.13154. Epub 2018 Feb 23.

12. Gardner C. Non-nutritive sweeteners: evidence for benefit vs. risk. Curr Opin Lipidol. 2014;25(1):80-4. doi: 10.1097/MOL.0000000000000034.

13. Grech A, Kam CO, Gemming L, et al. Diet-Quality and Socio-Demographic Factors Associated with NonNutritive Sweetener Use in the Australian Population. Nutrients. 2018;10(7):833. doi: 10.3390/nu10070833.

14. Lohner S, Toews I, Meerpohl JJ. Health outcomes of non- nutritive sweeteners: analysis of the research landscape. Nutr J. 2017;16(1):55. doi: 10.1186/s12937-017-0278-x.

15. Pepino MY. Metabolic effects of non-nutritive sweeteners. Physiol Behav. 2015;152:450-455. doi: 10.1016/j.physbeh.2015.06.024. Epub 2015 Jun 19.

16. Olalde-Mendoza L, Moreno-González YE. Modificación de la glucemia en ayuno en adultos con diabetes mellitus tipo 2 después de la ingesta de refrescos de cola y de dieta en el estado de querétaro, México [Modification of fasting blood glucose in adults with diabetes mellitus type 2 after regular soda and diet soda intake in the State of Querétaro, Mexico]. Arch Latinoam Nutr. 2013;63(2):142-147.

17. Hollrah D, Dawson K, Kennedye $P$, et al. Question: In patients with type 2 diabetes, does diet soda consumption contribute to decline in blood sugar control?. J Okla State Med Assoc. 2017;110(4):206-207.

18. Fenwick EK, Gan AT, Man RE, et al. Diet soft drink is associated with increased odds of proliferative diabetic retinopathy. Clin Exp Ophthalmol. 2018;46(7):767-776. doi:10.1111/ceo.13154

19. Sujanitha V, Sivansuthan S, Selvakaran $P$, et al. Overweight, obesity and chronic complications of diabetes mellitus in patients attending Diabetic Centre, Teaching Hospital, Jaffna, Sri Lanka. Ceylon Med J. 2015;60(3):94-96. doi:10.4038/cmj.v60i3.8065

20. Gray N, Picone G, Sloan F, et al. Relation between BMI and diabetes mellitus and its complications among US older adults. South Med J. 2015;108(1):29-36. doi:10.14423/SMJ.0000000000000214

21. Voigt M, Schmidt S, Lehmann T, et al. Prevalence and Progression Rate of Diabetic Retinopathy in Type 2 Diabetes Patients in Correlation with the Duration of Diabetes. Exp Clin Endocrinol Diabetes. 2018;126(9):570-576. doi:10.1055/s-0043-120570

22. Jenchitr $W$, Samaiporn $S$, Lertmeemongkolchai $P$, et al. Prevalence of diabetic retinopathy in relation to duration of diabetes mellitus in community hospitals of Lampang. J Med Assoc Thai. 2004;87(11):1321-1326.

23. Wat N, Wong RL, Wong IY. Associations between diabetic retinopathy and systemic risk factors. Hong Kong Med J. 2016;22(6):589-599. doi:10.12809/hkmj164869

\section{ACKNOWLEDGEMENTS}

We would like to acknowledge the Deanship of Higher Education and Scientific Research of the University of Tabuk for the financial support of this research under the grant (S1440-81) 


\section{PEER REVIEW}

Not commissioned. Externally peer reviewed.

\section{CONFLICTS OF INTEREST}

The authors declare that they have no competing interests.

\section{FUNDING}

The research is funded by the Deanship of Higher Education and Scientific Research of the University of Tabuk, grant number, S-1440-81.

\section{Table 1: The characters of the study group}

\begin{tabular}{|l|l|}
\hline Age (25-94) & $49.80 \pm 13.45$ \\
\hline Duration of diabetes & $9.36 \pm 6.56$ \\
\hline BMI & $26.96 \pm 4.51$ \\
\hline HbA $1_{c}$ & $8.50 \pm 1.60$ \\
\hline Soda soft drinks /week & $1.18 \pm 1.57$ \\
\hline Artificial sweeteners /day & $0.89 \pm 1.26$ \\
\hline Sex & \\
Males & $88(50.6 \%)$ \\
Females & $86(49.4 \%)$ \\
\hline BMI & \\
Obese & $36(20.7 \%)$ \\
overweight & $80(45.9 \%)$ \\
\hline Exercise & $86(49.4 \%)$ \\
\hline Family history of diabetes & $124(71.3 \%)$ \\
\hline Family history of hypertension & $96(55.2 \%)$ \\
\hline Family history of coronary syndrome & $26(14.9 \%)$ \\
\hline Hypertension drugs & $66(37.9 \%)$ \\
\hline Lipid-lowering medications & $40(23 \%)$ \\
\hline Diabetes medications & \\
Oral therapy & $62(35.6 \%)$ \\
\hline Insulin & $22(12.6 \%)$ \\
Both & $90(51.7 \%)$ \\
\hline Retinopathy (NON-PDR, PDR) & $54(31 \%)$ \\
\hline Diet soda consumers & $82(47.1 \%)$ \\
\hline
\end{tabular}

Table 2: The association between diabetic retinopathy, soda soft drinks, artificial sweeteners, BMI, duration of diabetes mellitus, and gender

\begin{tabular}{|l|l|l|l|}
\hline Character & Wald & P-value & $95 \% \mathrm{Cl}$ \\
\hline Soda soft drinks & 1.251 & 0.263 & $0.583-7.204$ \\
\hline Artificial sweeteners & 0.213 & 0.644 & $0.190-2.793$ \\
\hline BMI & 2.459 & 0.117 & $0.776-1.029$ \\
\hline Duration of diabetes & 8.095 & 0.004 & $0.766-0.952$ \\
\hline Gender & 0.223 & 0.637 & $0.369-5.092$ \\
\hline Constant & 4.691 & 0.030 & \\
\hline
\end{tabular}

